\title{
Fabrications of Terahertz Wave Resonators in Micro Liquid Cells Introduced into Alumina Photonic Crystals with Diamond Structures
}

\author{
Soshu Kirihara, Noritoshi Ohta, Toshiki Niki, and Satoko Tasaki \\ Joining and Welding Research Institute, Osaka University, 11-1 Mihogaoka Ibaraki, 567-0047 Osaka, Japan \\ Correspondence should be addressed to Soshu Kirihara, kirihara@jwri.osaka-u.ac.jp
}

Received 30 March 2011; Accepted 4 May 2011

Academic Editors: R. M. De La Cruz, V. Ralchenko, and V. Sglavo

Copyright (C) 2011 Soshu Kirihara et al. This is an open access article distributed under the Creative Commons Attribution License, which permits unrestricted use, distribution, and reproduction in any medium, provided the original work is properly cited.

\begin{abstract}
Terahertz wave resonators composed of three-dimensional photonic crystals composed of alumina lattices with diamond structures were designed and fabricated by using microstereolithography process. These three dimensional periodic structures of ceramics can reflect perfectly the terahertz waves through Bragg diffraction. A micro glass cell including water solvents was put between two photonic crystals to realize multiple resonations of terahertz waves to synchronize with various vibration modes of saccharide or protein molecules and control biochemical reactions through electromagnetic excitations.
\end{abstract}

\section{Introduction}

Photonic crystals with periodic arrangements of dielectric materials form photonic band gaps to totally reflect electromagnetic waves through Bragg diffraction $[1,2]$. By introducing an artificial defect of air cavities into the periodic arrangement, localized modes can be formed in the band gaps. These structural defects can localize and amplify electromagnetic waves at the specific frequencies and wavelengths corresponding to the sizes and dielectric constants of the resonation domains. For the last several decades, the photonic crystal has been expected to be applied for various microwave devices of resonators, filters, and directional antennas $[3,4]$. In our investigation group, micrometer order ceramic lattices with diamond structures were fabricated successfully by using microstereolithography system [5]. The diamond structures can create perfect photonic band gaps to reflect electromagnetic waves totally for all directions.

In recent years, the microwave waves in a terahertz frequency range have attracted considerable attentions as novel analytical light sources. Because the electromagnetic wave frequencies from 0.1 to $10 \mathrm{THz}$ can be synchronized with collective vibration modes of saccharide or protein molecules, the terahertz wave spectroscopy are expected to be applied to various types of sensors for detecting harmful substances in human blood, cancer cells in the skins, and micro bacteria in vegetables [6-10]. Moreover, the terahertz sensing technologies for aqueous phase environments in nature fields to detecting dissolved matters directly are extremely interesting topics. However, the terahertz waves are difficult to transmit into the water solvents with submillimeter order thickness corresponding to the several wavelengths due to electromagnetic absorptions [11].

In the previous investigations of other research groups, Fabry-perot cavities have been applied for terahertz wave resonators. However, it is theoretically unfeasible to achieve the effective single mode operation in the micro Fabry-perot resonators, because electromagnetic losses are in inverse proportion to the cavity size [12]. Compare with this, photonic crystals can realize the single mode resonation without the electromagnetic losses through the appropriate structural design. Especially, the three dimensional diamond lattices are regarded as the ideal photonic crystal structures. Figures 1(a) and 1(b) show a unit cell of the diamond photonic crystal and an electromagnetic band diagram calculated by using plane wave expansion method simulator (RSOFT Design Group Co. Ltd., USA; BandSOLVE), respectively [13]. The artificial crystal can totally reflect the terahertz wave with the corresponding wavelength to the lattice spacing through 
the Bragg diffraction. The complete photonic band gap is formed to prohibit electromagnetic wave expansions for all crystal directions. In the recent investigations, the diamond structures introduced a point or plane defect were formed successfully, and localized modes with several wavelengths were observed in the cavity regions $[14,15]$. From these obtained results, the diamond photonic crystals can be applied to low loss terahertz wave resonator.

In this investigation, we designed and fabricated the terahertz wave resonators composed of the defects introduced photonic crystals composed of the micrometer order alumina lattices with the diamond structures to resonate and localized the terahertz waves strongly into the water solvents in order to realize the higher analytical precisions. In the photonic crystal resonator, a micro glass cell including the water solvent was put between two diamond lattice structures as a plane defect. The micro structures of the dielectric lattice and resonation cavity modeled theoretically as described in Section 2. Subsequently, the real resonator module was fabricated successfully by using the micro stereolithographic procedure as described in Section 3. The incident terahertz wave was reflected multiply to achieve longer optical distance in the liquid region and localized to amplify the sensing signal in the transmission spectrum. In the fourth section, the terahertz wave properties of the resonation profiles in the transmission spectra and the localized modes in the plane defects through fabricated resonators were observed and visualized successfully by using a time domain spectroscopy and a finite difference time domain simulator, respectively.

\section{Theoretical Modeling}

The electromagnetic band properties of the diamond photonic crystals were calculated theoretically to determine the geometric parameters by using the plane wave expansion method [16]. Figure 2 shows the variations of complete band gap widths as the function of aspect ratio in the dielectric lattice. In the calculation, the alumina ceramics of 9.8 in dielectric constant was assumed as the lattice material. The aspect ratio was optimized as 1.5 to create the wider band gap. Subsequently, the gap frequency can be shifted for the lower range according in inverse proportion to the lattice spacing as shown in Figure 3. To open the band gapes in the terahertz frequencies, micro scale structural periods need to be created. The lattice constant was designed as $375 \mu \mathrm{m}$ corresponding to the band gap frequencies from 0.3 to $0.6 \mathrm{THz}$.

Resonance efficiencies of the terahertz wave resonator with the diamond lattices were optimized by using transmission line modeling simulator (Flomerics Co. Ltd., UK; Micro-Stripes Ver. 7.5) of a finite difference time domain method. Figure 4 shows a computer graphic model of the terahertz wave resonator. The micro glass cell including the water was sandwiched as the plane defect between two alumina photonic crystals with the diamond lattice. The defect thickness of the resonator and the period numbers of the diamond units were selected as the principle parameters to control the resonance characteristics. The defect thickness enables to tune the resonance frequencies in the band gap as shown in Figure 5. In this investigation, the plane defect was designed as the water cell of $470 \mu \mathrm{m}$ in thickness composed of two quartz plates of $160 \mu \mathrm{m}$ and an aqueous cavity of $150 \mu \mathrm{m}$. The period numbers of the diamond lattices enables to adjust the resonance qualities as shown in Figure 6. The resonance qualities can be enhanced by increasing the period numbers; however, the localized mode of the transmission peak becomes lower through the perfect confinement of the electromagnetic wave in the defect domain. The diamond lattices composed of two units in period number were optimized and designed in order to detect the sharp localized mode peak in the transmission spectrum.

\section{Experimental Procedure}

The photonic crystal resonators of the terahertz wave were fabricated by using computer-aided design and manufacturing processes. The diamond lattices were designed by using a computer graphic software (Toyota Caelum Co. Ltd., Japan; Think-Design Ver. 9.0). The lattice constant of the diamond structure and the aspect ratio of the dielectric lattice were $500 \mu \mathrm{m}$ and 1.5 , respectively. The whole size of the crystal component was $5 \times 5 \times 1 \mathrm{~mm}$ consisting of 10 $\times 10 \times 2$ unit cells. The designed model was converted into stereolithographic files of a rapid prototyping format and sliced into a series of two-dimensional cross-sectional data of $15 \mu \mathrm{m}$ in layer thickness. These data were transferred into an microstereolithographic equipment (D-MEC Co. Ltd., Japan; SI-C 1000). Figure 7 shows a schematic illustration of the microstereolithography system. Photosensitive acrylic resins including alumina particles of $170 \mathrm{~nm}$ in average diameter at $40 \%$ in volume content were supplied on a glass substrate from a dispenser nozzle by the air pressure. This paste was spread uniformly by using a mechanically controlled knife edge. The thickness of each layer was set at $15 \mu \mathrm{m}$. Two dimensional solid patterns are obtained by a light induced photo polymerization. High-resolution image has been achieved by using a digital micro mirror device. In this optical device, micro aluminum mirrors of $14 \mu \mathrm{m}$ in edge length were assembled with $1024 \times 768$ in numbers. Each mirror can be tilted independently by piezoelectric actuating. Through the layer-by-layer stacking under the computer control, the acrylic resin component with the alumina particles dispersion was obtained. The composite precursor was dewaxed at $600^{\circ} \mathrm{C}$ for $2 \mathrm{hs}$ and sintered at $1500^{\circ} \mathrm{C}$ for $2 \mathrm{hs}$ in the air atmosphere. Subsequently, in order to obtain a plane defect between the two diamond structures, a micro glass cell was also fabricated by using the microstereolithography. Figure 8(a) shows the schematically illustrated components of the resonance cells. The quartz plates of $160 \mu \mathrm{m}$ in thickness were inserted into the photosensitive acrylic resins in the stacking and exposing process. Finally, the micro resonators cell was put between the diamond photonic crystals, and the terahertz wave resonator was integrated successfully by using acrylic resin flames as shown in Figure 8(b). These flames were glued together by using the photosensitive liquid resin and the ultraviolet exposure solidification. Water solutions were infused through catheters connected on the top side of the resonance cell. 


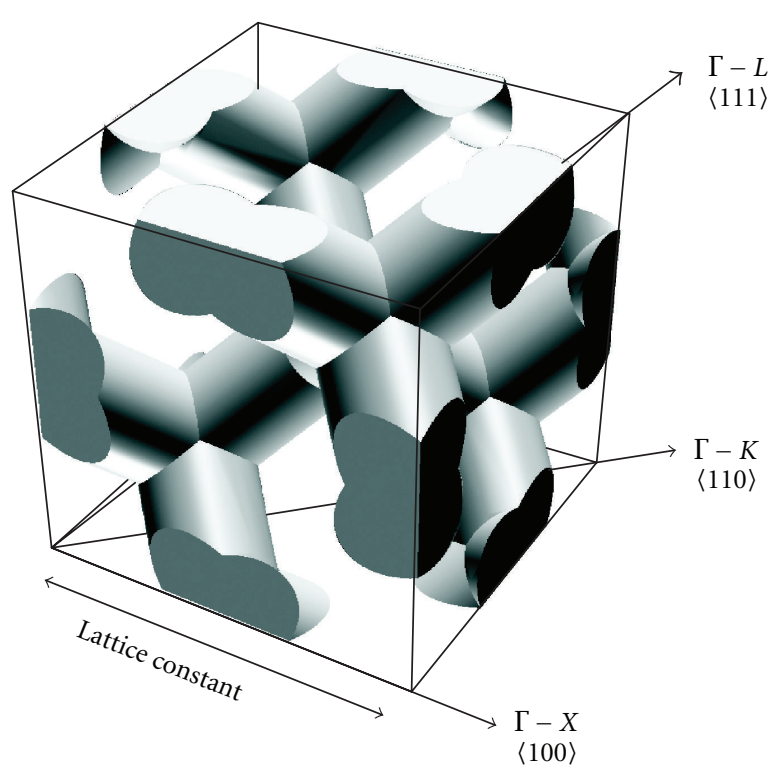

(a)

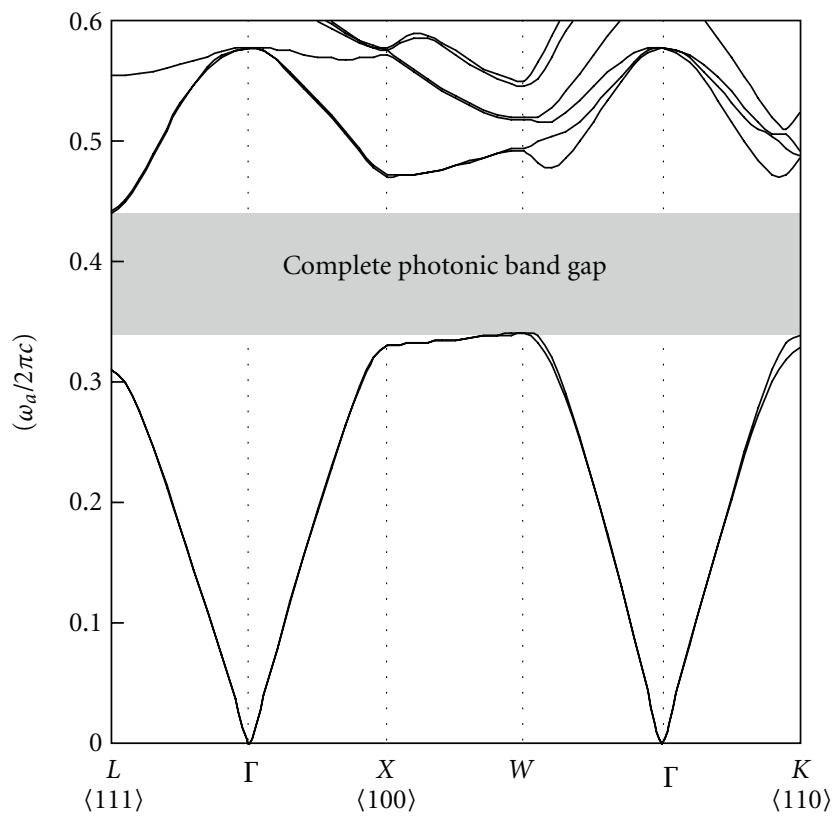

(b)

FIgUre 1: A CAD image of a unit cell of diamond structure (a) and a photonic band diagram of diamond lattice structure calculated by plane wave expansion (PWE) method (b).

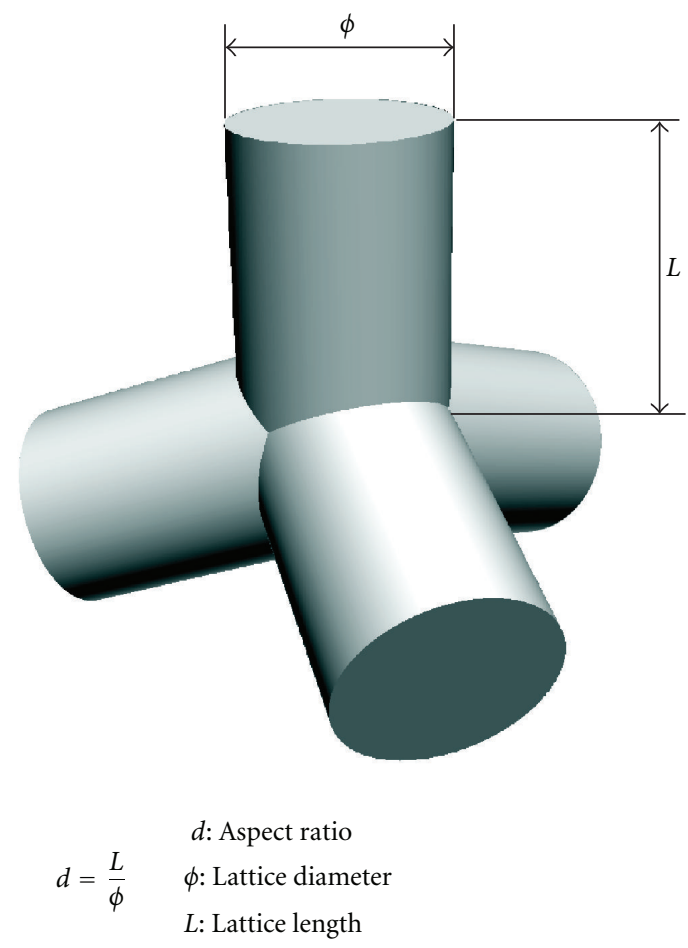

(a)

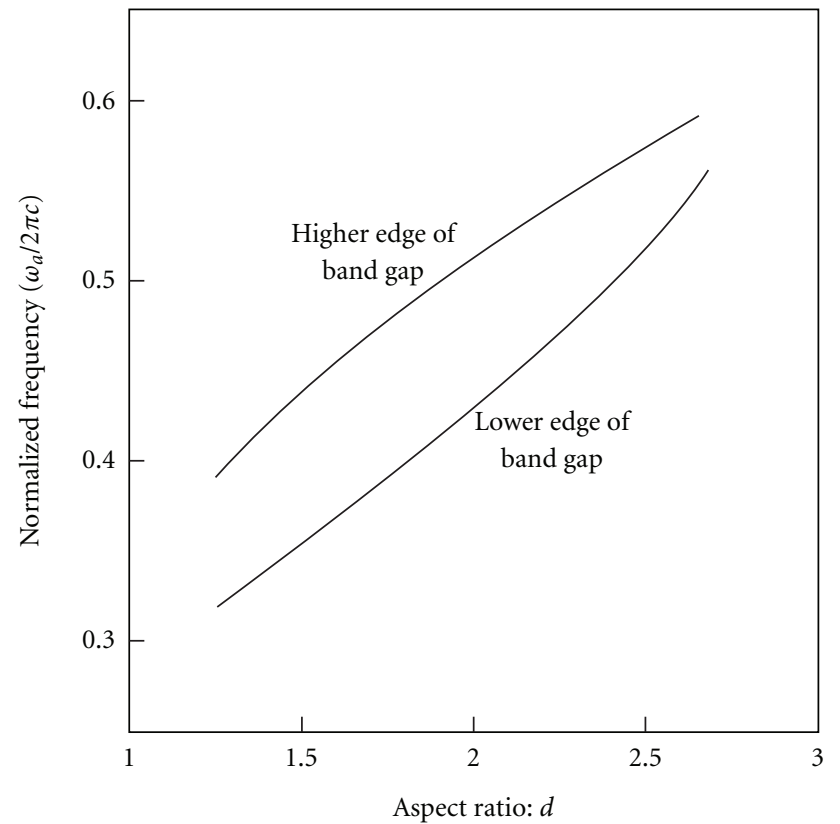

(b)

FIGURE 2: Definition of aspect ratio of diamond structure (a) and band gap width as a function of aspect of aspect ratio (b). 


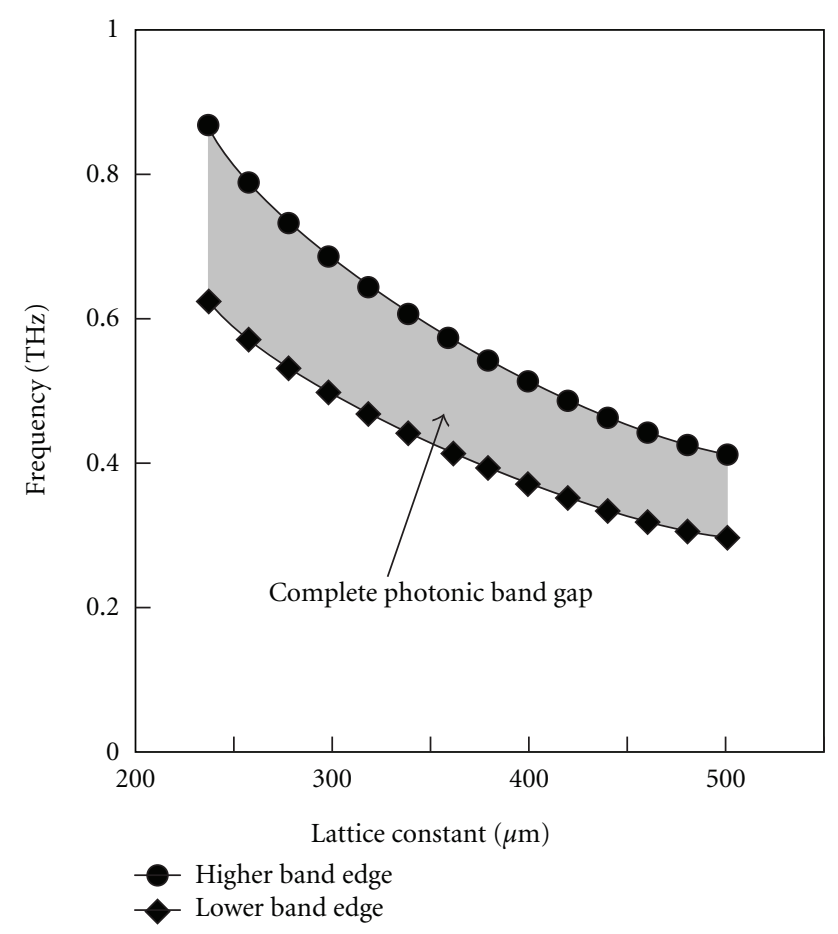

FIGURE 3: Variations of electromagnetic bands as functions of lattice constants in diamond structures.

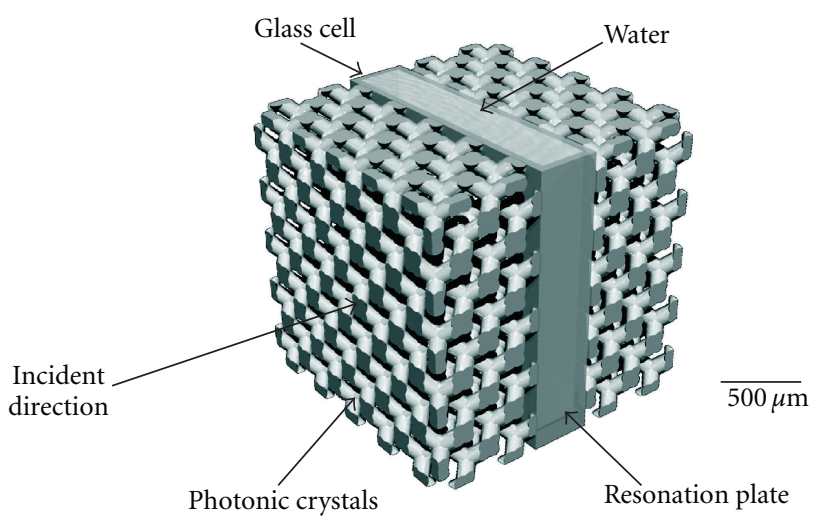

Figure 4: A computer graphic model of a terahertz wave micro reactor.

The transmission properties of incident terahertz waves were analyzed by using the terahertz time domain spectroscopy (Advanced Infrared Spectroscopy Co. Ltd., Japan, J-spec $2001 \mathrm{spc} / \mathrm{ou})$. The distributions of electric field intensities in the resonator were simulated and visualized at the localized frequency by using the transmission line modeling.

\section{Results and Discussion}

Figure 9(a) shows the photonic crystal composed of the acryl lattice including with the alumina particles formed by the microstereolithography. Tolerance between the designed and formed object was successfully converged within $5 \mu \mathrm{m}$.

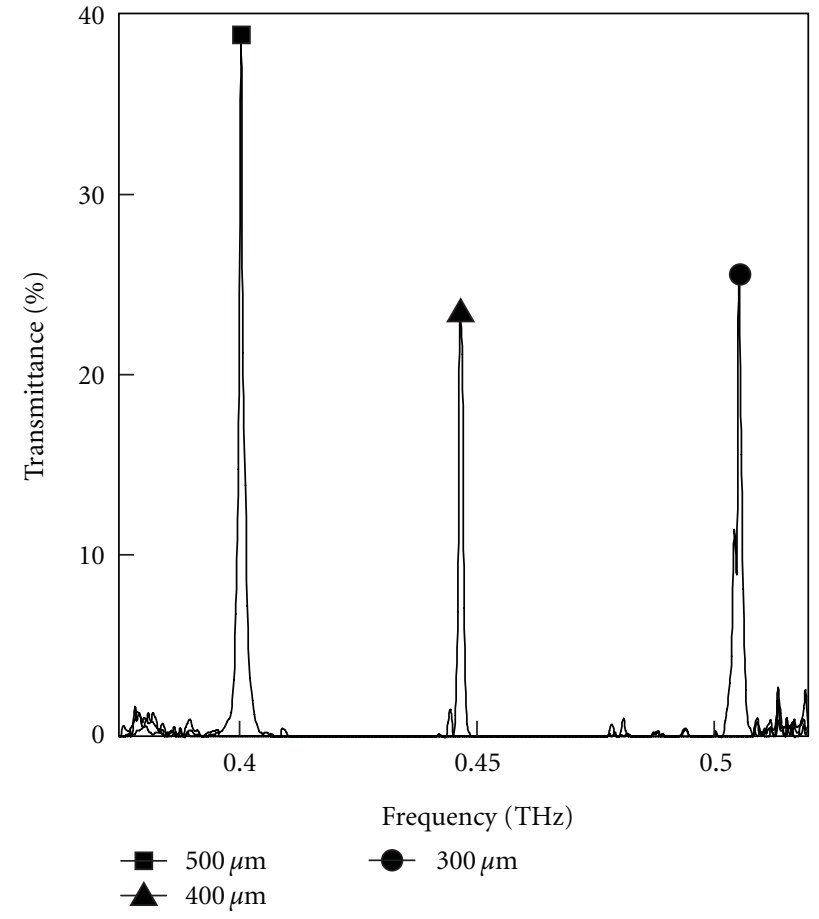

FIGURE 5: Calculated localized mode formations through electromagnetic waves resonations in micro cells with various thicknesses.

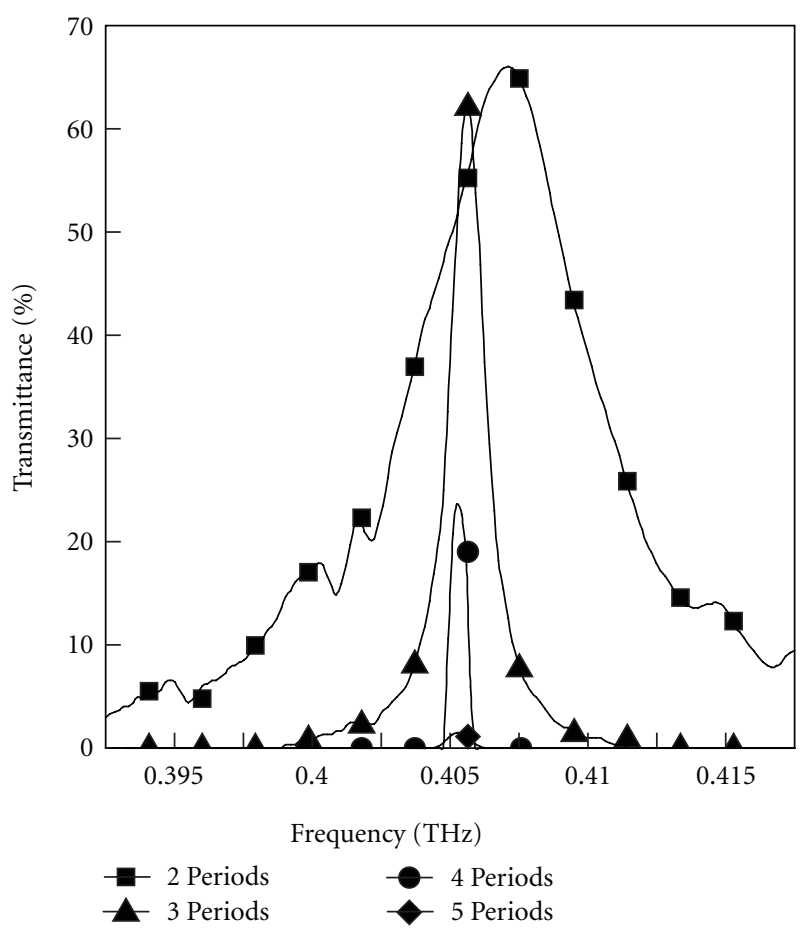

FIGURE 6: Calculated peak profiles of multiple resonation modes in water cells of plane defects put between photonic crystals with various layer numbers. 


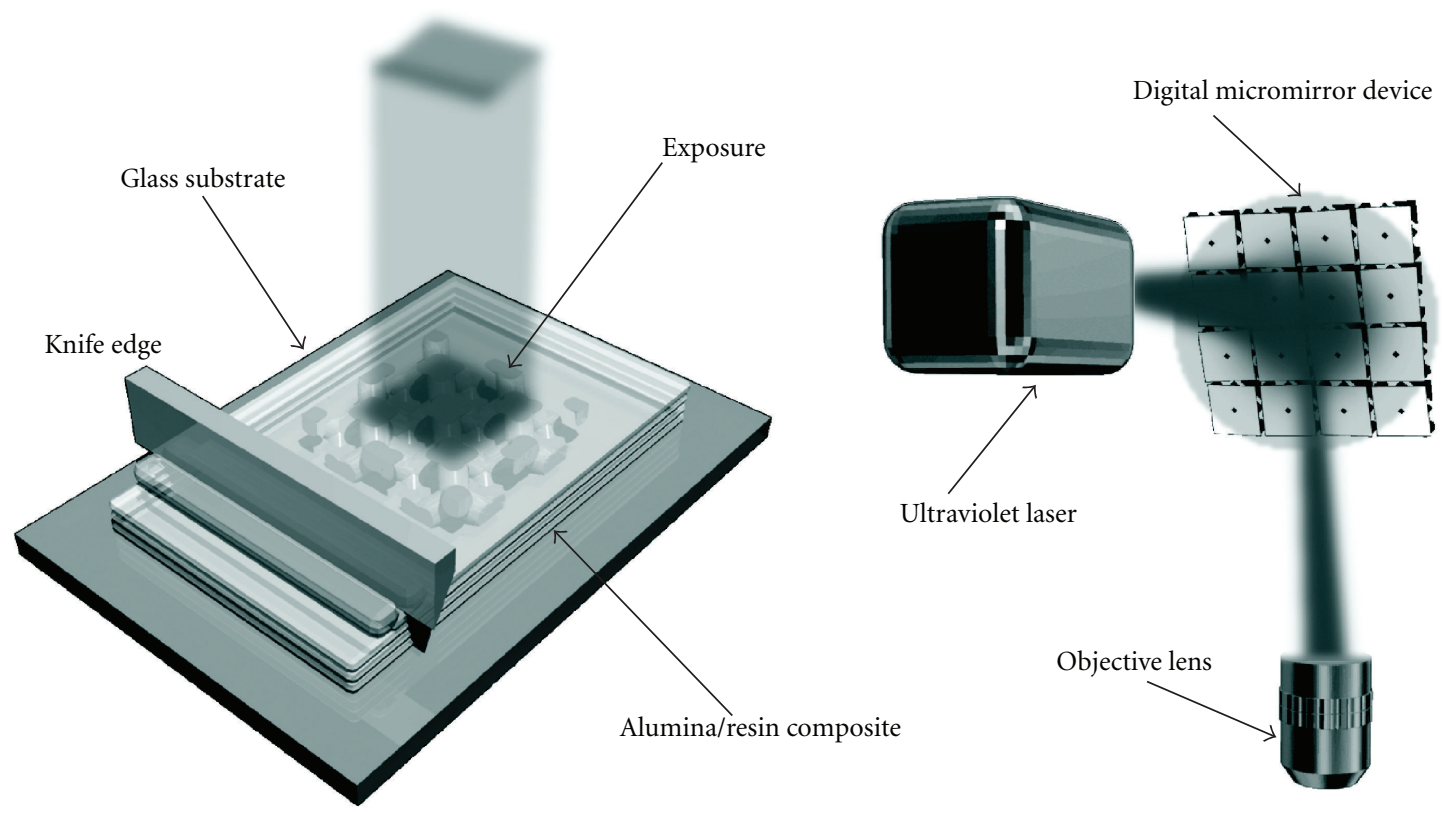

FIGURE 7: A schematically illustrated microstereolithography system.

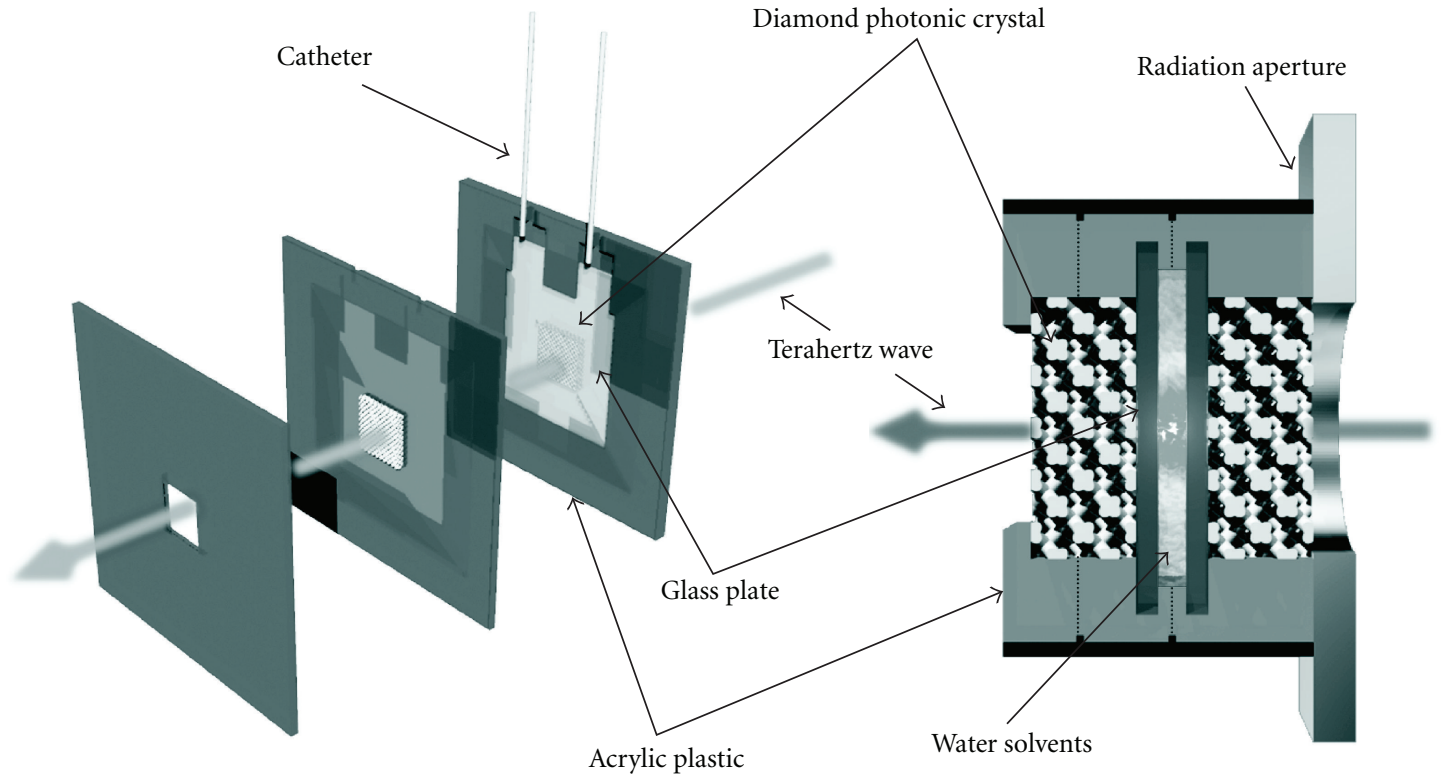

(a)

(b)

Figure 8: A schematic illustration of the parts of the resonance cells (a) and fabricated $\mathrm{THz}$ wave resonator (b). The resonator was successfully created by integrating the diamond structures into the cells to realize multiple resonations of the THz wave in aqueous phase.

Figure 9(b) shows the alumina lattice with the diamond structure fabricated by ceramic powder sintering. No cracks or deformations were observed in the obtained components. The average linear shrinkage was $25 \%$. The lattice constant of the sintered sample was $375 \mu \mathrm{m}$. Figure 9(c) shows the microstructures of the sintered sample observed by a scanning electron microscope. The relative density of the sample reached $97.5 \%$.
The integrated terahertz wave resonator is shown in Figure 10. The two diamond lattice components were attached on the quartz glasses, and these two glass plates were arranged with $150 \mu \mathrm{m}$ in parallel interval. The tolerance for the transmission direction of the electromagnetic wave was converged within $5 \mu \mathrm{m}$. The cell capacity was $0.02 \mathrm{~mL}$. Figure 11(a) shows the measured transmission spectra for the resonators. Distilled water or ethanol was infused into the 


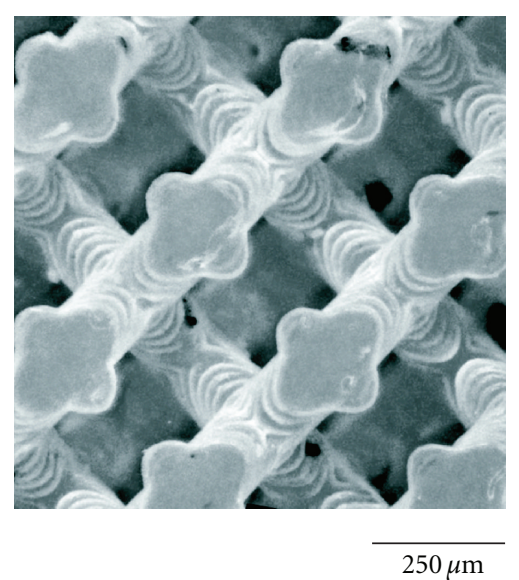

(a)

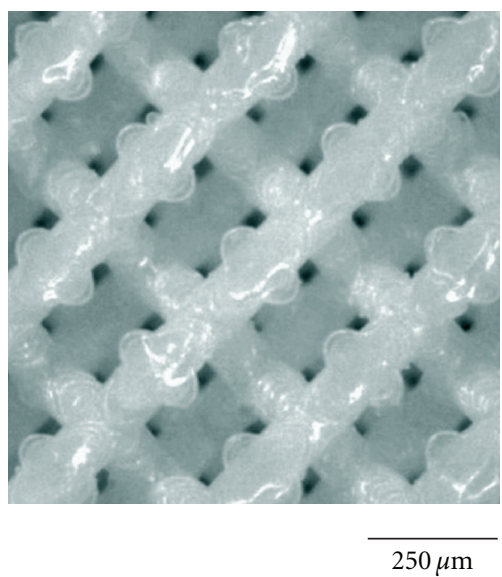

(b)

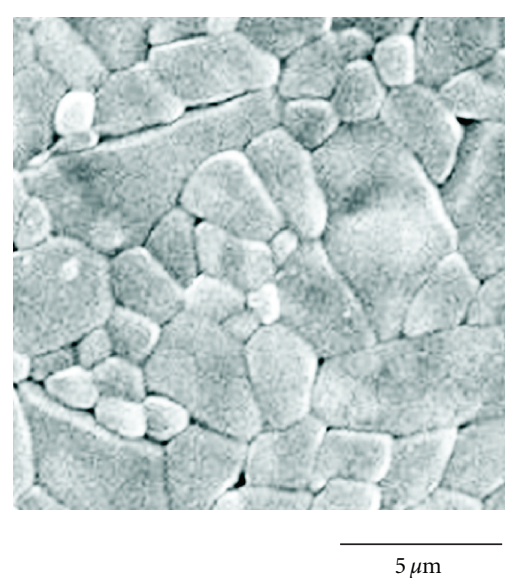

(c)

FIGURE 9: A diamond-type acryl lattice with alumina particles dispersions formed by the micro-stereolithography (a), a sintered alumina photonic crystals (b), and an SEM micrograph of the sintered alumina lattice (c).

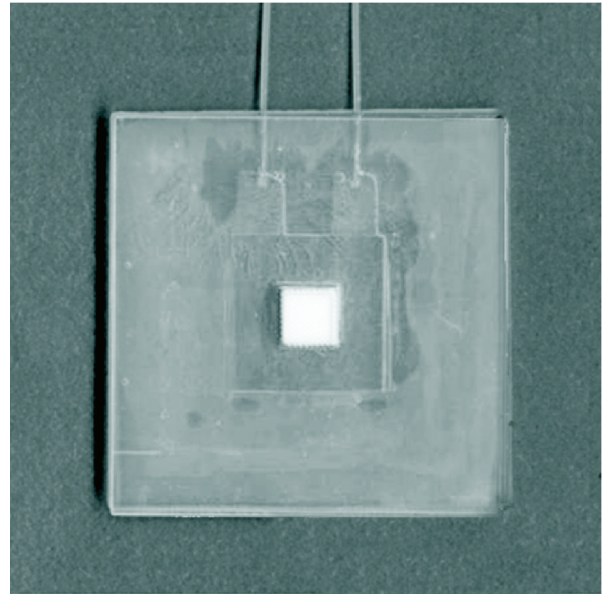

$1 \mathrm{~mm}$

(a)

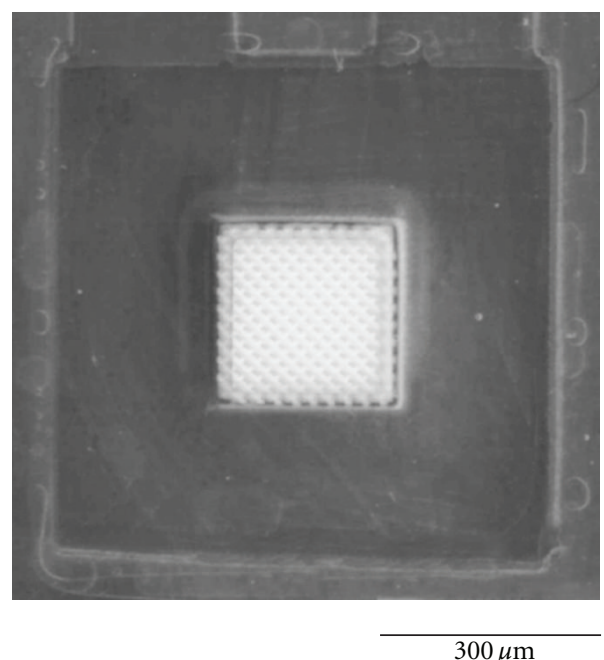

(b)

FIGURE 10: A terahertz wave resonator with micro liquid cell sandwiched between diamond photonic crystals. Distilled water and ethanol were infused through the catheters implanting in the top side of the cells, respectively.

micro cells. In the case of distilled water, two localized modes of transmission peaks were observed at 0.410 and $0.491 \mathrm{THz}$ in frequencies in the photonic band gap. In the case of ethanol, an amplification peak was observed at $0.430 \mathrm{THz}$. The measured band gap ranges and the localized mode frequencies have good agreement with the simulated results by the transmission line modeling as shown in Figure 11(b). In the transmission spectrum through the photonic crystal resonator including the water, the localized modes of the higher and lower peak frequencies are defined modes A and $\mathrm{B}$, respectively. And the localized mode peak in the transmission spectrum through the ethanol is defined mode C. The cross sectional profiles of the electric field intensity corresponding to the localized modes $\mathrm{A}, \mathrm{B}$, and $\mathrm{C}$ were simulated and visualized theoretically as shown in Figures 12(a), 12(b), and 12(c), respectively. The terahertz wave was propagated from the left to the right side. The white and black areas show that the electric field intensity is high and low, respectively. The incident terahertz waves were resonated and localized strongly through the multiple reflections in the liquid cell between two diffraction lattices with the diamond structures. Concerning with the localized mode A as shown in Figure 12(a), the standing wave with five nodes was observed in the vicinity area of the glass cell. And the localized mode B with the lower frequency and the longer wavelength is composed of the standing wave with four nodes between the diffraction lattices as shown in Figure 12(b). Concerning with the localized mode $C$ in the ethanol as shown in Figure 12(c), the standing wave with four nodes has the similar resonance profiles between the diffraction lattices as the localized mode B in the water. Figure 12(d) shows the dielectric constants and composite dimensions 


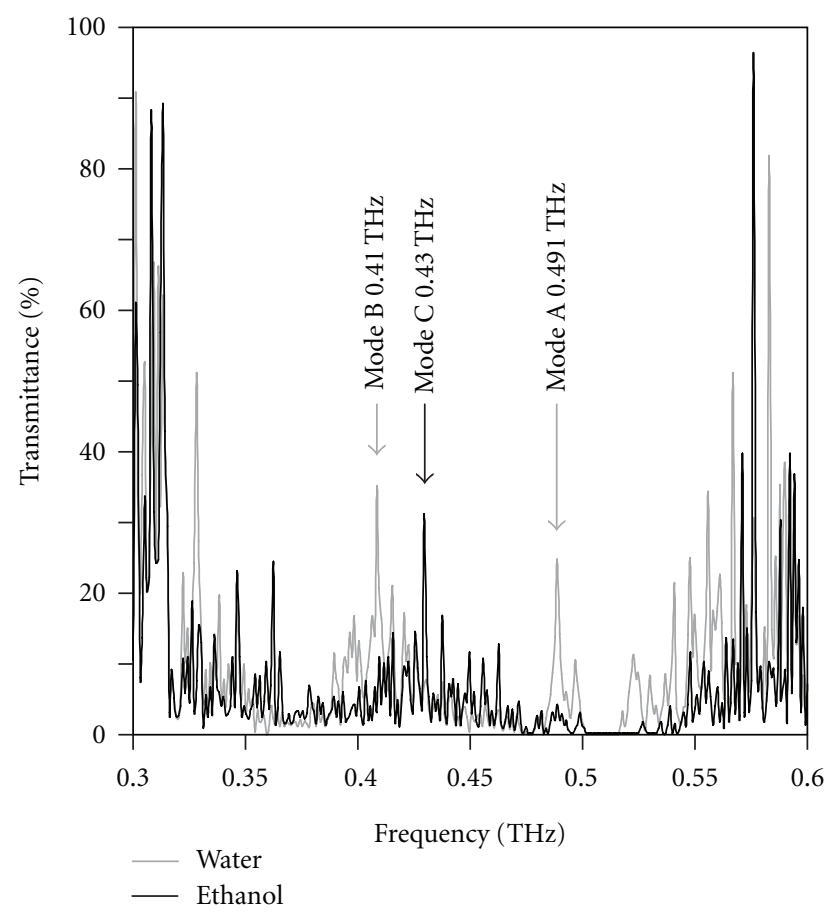

(a)

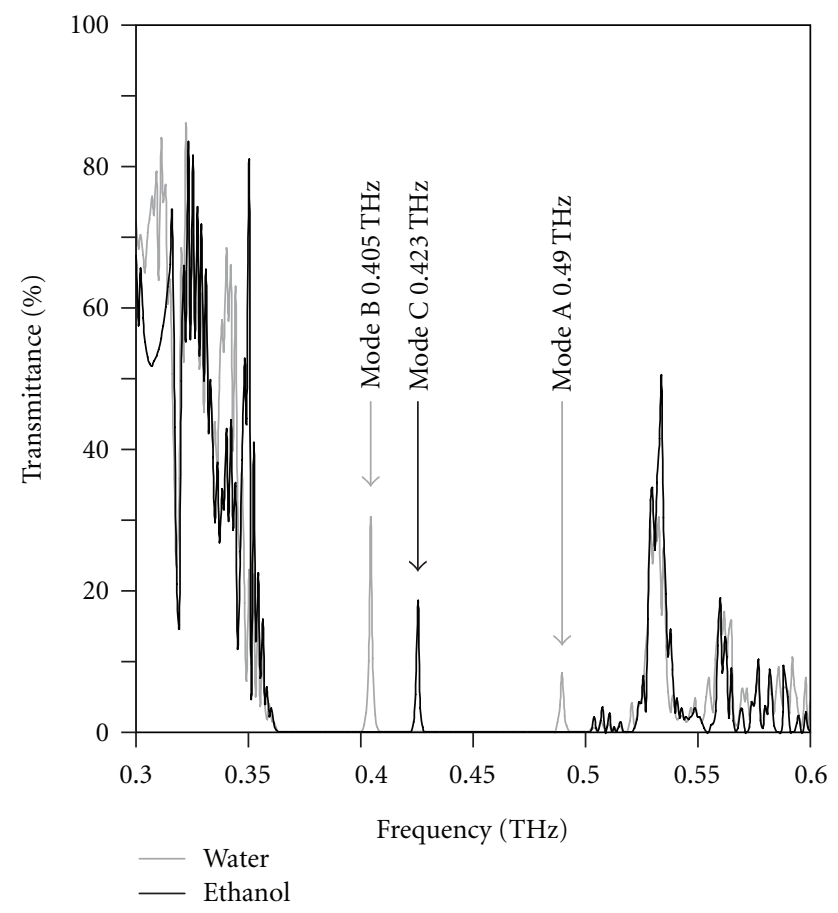

(b)

Figure 11: Transmission spectra for the resonators infusing distilled water and ethanol. The spectra (a) and (b) are measured and calculated properties by using the terahertz wave time domain spectroscopy and a transmission line modeling methods, respectively.
0

0.00025

0.0005

Electric field intensity $(\mathrm{V} / \mathrm{m})$

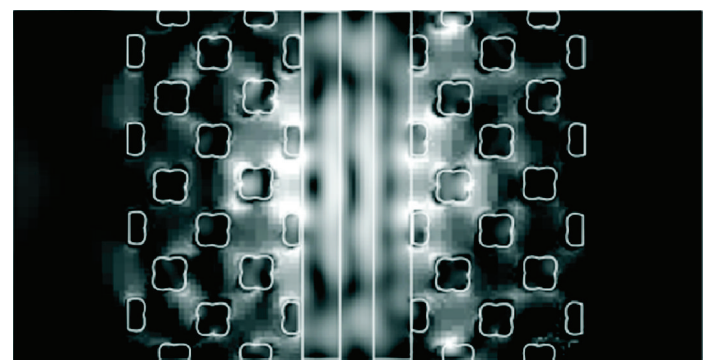

(a)

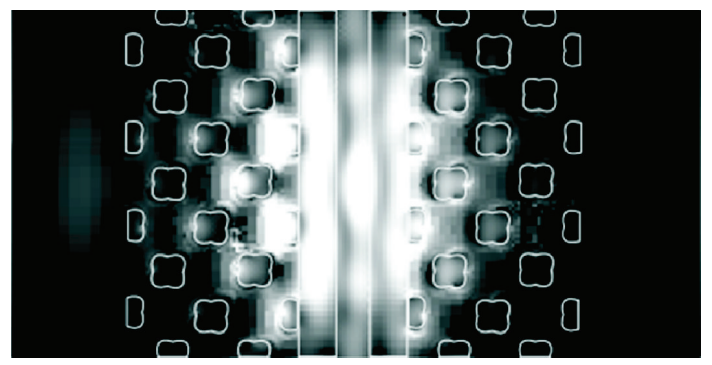

(b)

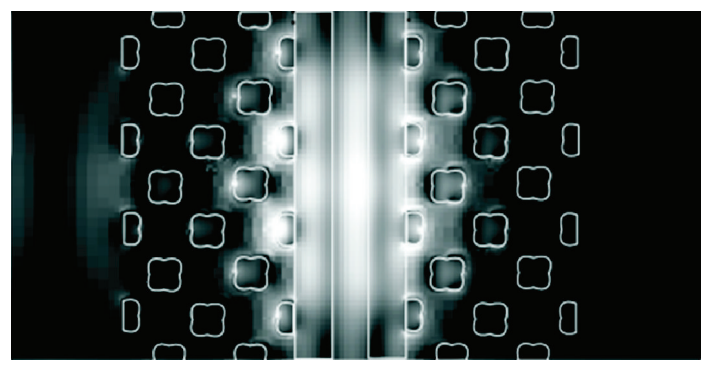

(c)

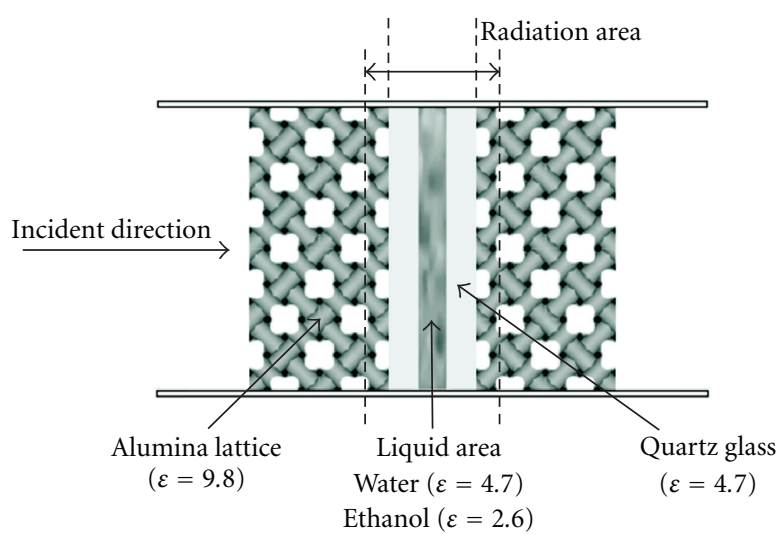

(d)

Figure 12: Electric field distributions of localized modes simulated by transmission line modeling (TLM) method of a finite difference time domain (FDTD). The localized modes A, B, and C, are shown in (a), (b), and (c), respectively. Dielectric constants and composite dimensions of the photonic crystal resonator are shown in $(\mathrm{d})$. 
of the photonic crystal resonator. The dielectric constants of water and ethanol are 4.7 and 2.6 in the terahertz wave frequency range $[17,18]$. By using the terahertz wave time domain spectroscopy, the dielectric constants of alumina and quartz grass were measured as 9.8 and 3.8. In comparison with the resonance areas of the localized modes B and C, the effective dielectric constants decrease from 3.02 to 2.59, and the optical lengths decrease from 1.13 to $1.05 \mathrm{~mm}$, respectively. Therefore, the resonance peak frequencies of the localized mode $\mathrm{B}$ and $\mathrm{C}$ with the similar standing wave profiles are shifted clearly from 0.410 to $0.430 \mathrm{THz}$ by replacing the water with ethanol in the resonator. Form these results, the fabricated phonic crystal resonator is considered to be a promising candidate as the novel analytical device to determine the dissolved components in the aqueous solution by using the terahertz spectroscopy.

\section{Conclusions}

In terahertz wave resonators, a micro glass cell was put between two photonic crystals composed of alumina lattices with a diamond structure. These components were fabricated successfully by using microstereolithography. Transmission spectra were measured through the photonic crystal resonators including pure water or ethanol. Localized modes of sharp transmission peaks were observed in the photonic band gaps. In a distribution profile of electric field intensity simulated by using transmission line modeling, the strong localized modes were formed through multiple reflections in the liquid regions between the diffraction lattices. Moreover, the localized mode peak was shifted clearly from higher to lower frequencies through replacing the pure water with the ethanol. The fabricated photonic crystal resonator is considered to be a promising candidate for novel analytical devices to detect the compositional variations in natural aqueous phase environments.

\section{Acknowledgment}

This paper was supported by Priority Assistance for the Formation of Worldwide Renowned Centers of ResearchThe Global COE Program (Project: Center of Excellence for Advanced Structural and Functional Materials Design) from the Ministry of Education, Culture, Sports, Science, and Technology (MEXT), Japan.

\section{References}

[1] E. Yablonovitch, "Inhibited spontaneous emission in solidstate physics and electronics," Physical Review Letters, vol. 58, no. 20, pp. 2059-2062, 1987.

[2] S. John, "Strong localization of photons in certain disordered dielectric superlattices," Physical Review Letters, vol. 58, no. 23, pp. 2486-2489, 1987.

[3] S. Noda, "Three-dimensional photonic crystals operating at optical wavelength region," Physica B, vol. 279, no. 1-3, pp. 142-149, 2000.
[4] S. Noda, K. Tomoda, N. Yamamoto, and A. Chutinan, "Full three-dimensional photonic bandgap crystals at near-infrared wavelengths," Science, vol. 289, no. 5479, pp. 604-606, 2000.

[5] W. Chen, S. Kirihara, and Y. Miyamoto, “Three-dimensional microphotonic crystals of $\mathrm{ZrO} 2$ toughened $\mathrm{Al} 2 \mathrm{O} 3$ for terahertz wave applications," Applied Physics Letters, vol. 91, no. 15, Article ID 153507, 2007.

[6] M. Yamaguchi, F. Miyamaru, K. Yamamoto, M. Tani, and M. Hangyo, "Terahertz absorption spectra of L-, D-, and DLalanine and their application to determination of enantiometric composition," Applied Physics Letters, vol. 86, no. 5, Article ID 053903, pp. 1-3, 2005.

[7] B. M. Fischer, M. Walther, and P. U. Jepsen, "Far-infrared vibrational modes of DNA components studied by terahertz time-domain spectroscopy," Physics in Medicine and Biology, vol. 47, no. 21, pp. 3807-3814, 2002.

[8] Y. Oyama, L. Zhen, T. Tanabe, and M. Kagaya, "Subterahertz imaging of defects in building blocks," NDT and E International, vol. 42, no. 1, pp. 28-33, 2009.

[9] V. P. Wallace, A. J. Fitzgerald, S. Shankar et al., "Terahertz pulsed imaging of basal cell carcinoma ex vivo and in vivo," British Journal of Dermatology, vol. 151, no. 2, pp. 424-432, 2004.

[10] M. Hineno and H. Yoshinaga, "Far-infrared spectra of mono, di- and tri-saccharides in $50-16 \mathrm{~cm}-1$ at liquid helium temperature," Spectrochimica Acta, vol. 30, no. 2, pp. 411-416, 1974.

[11] M. R. Kutteruf, C. M. Brown, L. K. Iwaki, M. B. Campbell, T. M. Korter, and M. J. Heilweil, "Terahertz spectroscopy of short-chain polypeptides," Chemical Physics Letters, vol. 375, no. 3-4, pp. 337-343, 2003.

[12] Y. Akahane, T. Asano, B. Song, and S. Noda, "High-Q photonic nanocavity in a two-dimensional photonic crystal," Nature, vol. 425, no. 6961, pp. 944-947, 2003.

[13] K. M. Ho, C. T. Chan, and C. M. Soukoulis, "Existence of a photonic gap in periodic dielectric structures," Physical Review Letters, vol. 65, no. 25, pp. 3152-3155, 1990.

[14] H. Kanaoka, S. Kirihara, and Y. Miyamoto, "Terahertz wave properties of alumina microphotonic crystals with a diamond structure," Journal of Materials Research, vol. 23, no. 4, pp. 1036-1041, 2008.

[15] S. Kirihara and Y. Miyamoto, "Terahertz wave control using ceramic photonic crystals with a diamond structure including plane defects fabricated by micro stereolithography," International Journal of Applied Ceramic Technology, vol. 6, no. 1, pp. 41-44, 2009.

[16] J. Haus, "A brief review of theoretical results for photonic band structures," Journal of Modern Optics, vol. 41, pp. 195-207, 1994.

[17] H. Yada, M. Nagai, and K. Tanaka, "The intermolecular stretching vibration mode in water isotopes investigated with broadband terahertz time-domain spectroscopy," Chemical Physics Letters, vol. 473, no. 4-6, pp. 279-283, 2009.

[18] Y. Yomogida, Y. Sato, R. Nozaki, T. Mishina, and J. Nakahara, "Dielectric study of normal alcohols with $\mathrm{THz}$ time-domain spectroscopy," Journal of Molecular Liquids, vol. 154, no. 1, pp. 31-35, 2010. 

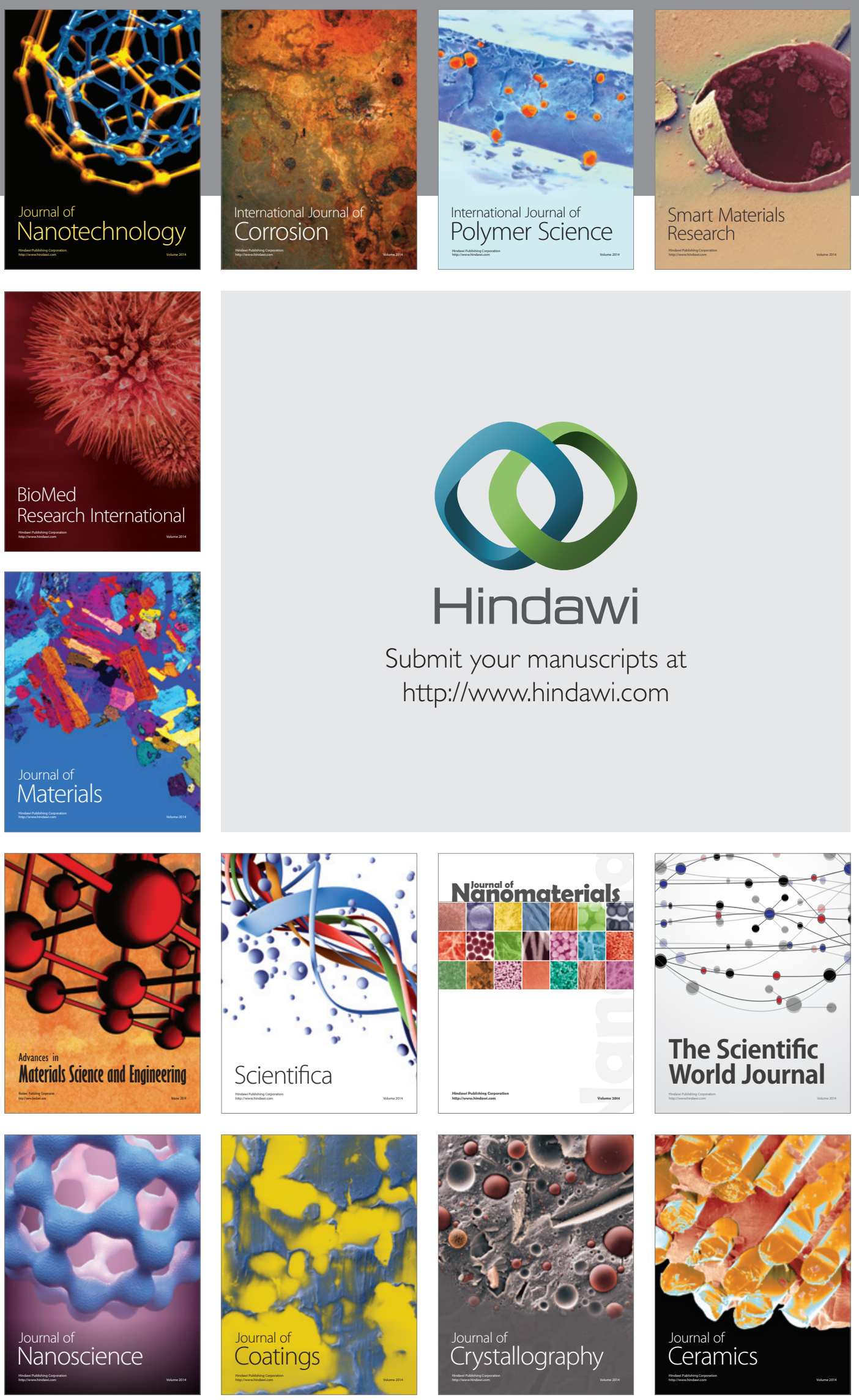

The Scientific World Journal

Submit your manuscripts at

http://www.hindawi.com

\section{World Journal}

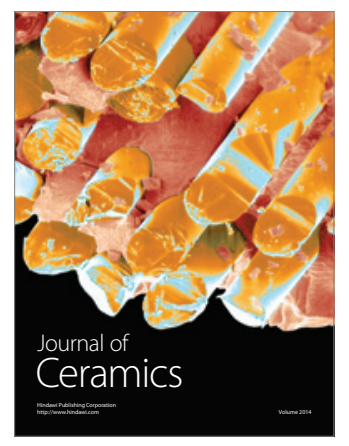

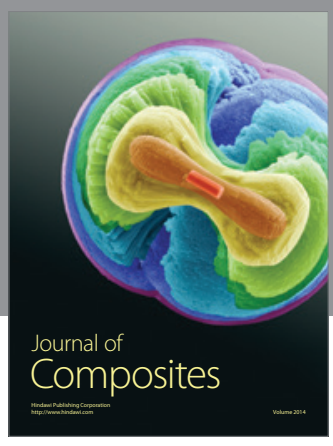
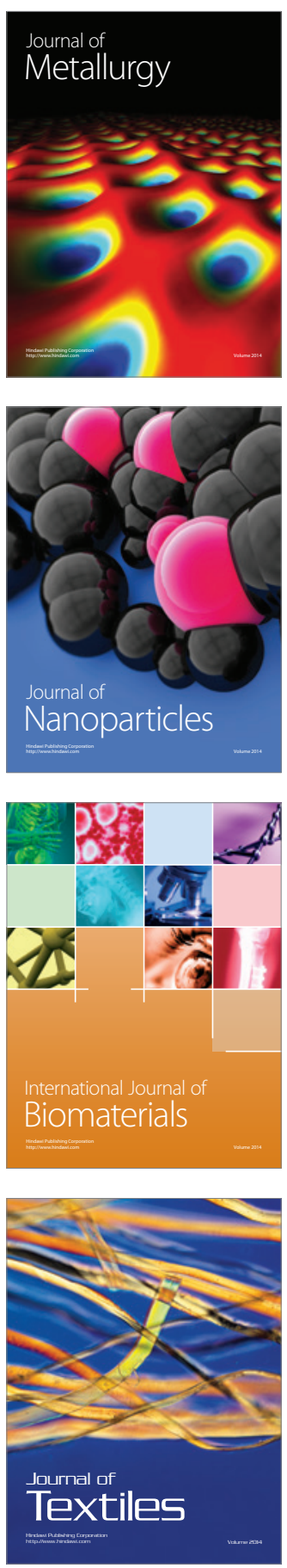